\title{
Granulocyte colony-stimulating factor does not promote neurogenesis after experimental intracerebral haemorrhage
}

\author{
Bernd Kallmünzer ${ }^{1 *}$, Miyuki Tauchi ${ }^{1,2}$, Johannes C. Schlachetzki², Kristin Machold'; \\ Ariana Schmidt'; Jürgen Winkler ${ }^{2}$, Stefan Schwab', and Rainer Kollmar ${ }^{1}$
}

\begin{abstract}
Background Hematopoietic growth factors have been suggested to induce neuroprotective and regenerative effects in various animal models of cerebral injury. However, the pathways involved remain widely unexplored.

Aims This study aimed to investigate effects of local and systemic administration of granulocyte colony-stimulating factor on brain damage, functional recovery, and cerebral neurogenesis in an intracerebral haemorrhage whole blood injection model in rats.

Methods Eight-week-old male Wistar rats $(n=100)$ underwent induction of striatal intracerebral haemorrhage by autologous whole blood injection or sham procedure and were randomly assigned to either (a) systemic treatment with granulocyte colony-stimulating factor $(60 \mu \mathrm{g} / \mathrm{kg})$ for five-days; (b) single intracerebral injection of granulocyte colonystimulating factor $(60 \mu \mathrm{g} / \mathrm{kg})$ into the cavity; or (c) application of vehicle for five-days. Bromodeoxyuridine-labelling and immunohistochemistry were used to analyze proliferation and survival of newly born cells in the sub-ventricular zone and the hippocampal dentate gyrus. Moreover, functional deficits and lesion volume were assessed until day $\mathbf{4 2}$ after intracerebral haemorrhage.

Results Differences in lesion size or hemispheric atrophy between granulocyte colony-stimulating factor-treated and control groups did not reach statistical significance. Neither systemic, nor local granulocyte colony-stimulating factor administration induced neurogenesis within the dentate gyrus or the sub-ventricular zone. The survival of newborn cells in these regions was prevented by intracerebral granulocyte colony-stimulating factor application. A subtle benefit in functional recovery at day 14 after intracerebral haemorrhage induction was observed after granulocyte colony-stimulating factor treatment.

Conclusion There was a lack of neuroprotective or neuroregenerative effects of granulocyte colony-stimulating factor in the present rodent model of intracerebral haemorrhage. Conflicting results from functional outcome assessment require further research.

Key words: acute stroke therapy, brain bleed, cerebral haemorrhage,
\end{abstract} neuroprotection, stem cells, stroke

Correspondence: Bernd Kallmünzer*, Department of Neurology, University Medical Centre Erlangen, Schwabachanlage 6, Erlangen 91054, Germany.

E-mail: bernd.kallmuenzer@uk-erlangen.de

${ }^{1}$ Department of Neurology, University Medical Center Erlangen, Germany

${ }^{2}$ Department of Molecular Neurology, University Medical Center Erlangen, Germany

Received: 30 August 2013; Accepted: 6 October 2013; Published online 19 December 2013

Conflict of interests: The authors report no conflicts of interest.

Funding: This study was supported by the ELAN-Fonds of the Medical Faculty, Friedrich-Alexander University Erlangen-Nürnberg, Germany.

DOI: $10.1111 /$ ijs.12217

\section{Introduction}

Intracerebral haemorrhage (ICH) accounts for $10-15 \%$ of all strokes and is associated with high mortality and severe disability (1). Evidence-based treatment strategies are currently limited to the prevention and management of secondary complications like hematoma expansion, hydrocephalus, and increased intracerebral pressure. However, innovative therapeutic approaches are presently under investigation, including the minimal invasive hematoma evacuation (NCT 00224770) and the treatment with neuroprotective or neuroregenerative agents (2). Granulocyte colony-stimulating factor (GCSF) as an endogenous hematopoietic growth factor, which crosses the blood brain barrier, binds to neuronal receptors, and improves functional outcome in different animal models of brain injury (3), is a promising candidate for ICH treatment. GCSF application has been shown to result in better recovery from functional deficits in an $\mathrm{ICH}$ animal model using intracerebral injection of bacterial collagenase $(4,5)$. Thereby, the proneurogenic effect of GCSF was repeatedly suggested as an underlying mechanism of GCSF action, but results are inconsistent and the pathways involved remain poorly understood at present $(3,5-7)$. The aim of this study was to investigate the previously described neuroprotective effects of GCSF treatment in a different animal model of $\mathrm{ICH}$ and to explore the role of GCSF on post-ICH neurogenesis. As modern surgical techniques permit the combination of hematoma evacuation with intracerebral injection of neuroprotectants, a group with intracerebral GCSF administration was included into the experimental setting and compared with systemic treatment.

\section{Methods}

\section{Animals and experimental procedures}

The study was conducted in accordance with the guide from the National Institute of Health for the care and use of laboratory animals, and the protocol was approved by local authorities for animal welfare. One hundred and four male Wistar Rats (Charles River, Sulzfeld, Germany, 10 weeks old, weight $348 \pm 22$ g) were housed singly with ad libitum food and water access. The experiments were performed in a blinded fashion. For hematoma induction, animals were anesthetized using isoflurane in $60 \%$ air and $40 \% \mathrm{O}_{2}(4-4 \cdot 5 \%$ for induction, $2-2 \cdot 5 \%$ during maintenance). ICH was induced using a modified whole blood injection method as described previously (8). Briefly, a burr hole was drilled above the right hemisphere at $3.5 \mathrm{~mm}$ lateral to bregma. A 26-G needle (Hamilton, Bonaduz, Switzerland) was inserted to a depth of $6 \mathrm{~mm}$ below the skull surface, and $70 \mu \mathrm{l}$ autologous 
blood was manually injected into the striatum. The injection was performed with continuous flow over a period of 10 mins and followed by a delay of 10 mins prior to needle removal. The burr hole was closed using bone wax, and the scalp was stapled. Xylocain $1 \%$ was applied for local anesthesia. Body temperature was continuously measured using a rectal probe and maintained constant at $37^{\circ} \mathrm{C}$ using a thermostatically controlled heating pad (Föhr Medical Instruments, Seeheim-Ober Beerbach, Germany). In animals subjected to the sham group, all procedures were performed in analog, however, without injection of blood. For euthanasia, the animal received deep anesthesia with sodium pentobarbital $(100 \mathrm{mg} / \mathrm{kg})$ and underwent transcardial perfusion with $\mathrm{NaCl} 0.9 \%$ followed by paraformaldehyde $4 \%$ in phosphatebuffered saline. Brains were removed and postfixed with $4 \%$ paraformaldehyde for $24 \mathrm{~h}$. Afterward, the brains were stored in $30 \%$ sucrose.

\section{Experimental groups and timeline}

Animals meeting one of the following criteria were excluded: (a) preexisting neurologic deficits (NDS $>0$ ) before ICH-surgery; (b) no measurable functional deficit on the first day after ICH surgery (NDS $=0$ ); and (c) very severe or fatal functional deficit on the first day after ICH surgery (NDS $>11$ ) leading to immediate euthanasia. A total of $n=4$ animals ( $4 \%$ ) were excluded $(n=3$ died during surgery, $n=1$ required euthanasia in the first hour after surgery due to fatal deficits). The remaining animals ( $n=100)$ were included and randomly assigned to one of the four groups: group I (systemic GCSF, $n=30$ ) received a daily intraperitoneal injection of $60 \mu \mathrm{g} / \mathrm{kg}$ GCSF (Amgen GmbH, Munich, Germany) dissolved in $1.0 \mathrm{ml}$ saline for five-days, starting $24 \mathrm{~h}$ after ICH induction. Group II (control, $n=26$ ) received $1 \mathrm{ml}$ saline via an intraperitoneal injection for five-days, starting $24 \mathrm{~h}$ after ICH induction. Group III (local GCSF, $n=27$ ) received a single injection of GCSF $(60 \mu \mathrm{g} / \mathrm{kg})$ into the cerebral hematoma just following its induction using the same burr hole. Animals of group IV (sham, $n=17$ ) were assigned to sham procedure without blood injection. The study was split into two parts (Fig. 1): animals in part one $(n=54)$ received intraperitoneal application of bromodeoxyuridine (BRDU) $(50 \mu \mathrm{g} / \mathrm{kg} /$ day $)$ from day 1 to day 5 after ICH and survived until day 7. In part two $(n=46)$, the animals were followed up until day 42 , whereas BRDU was administered on day 14 to day 18 .

\section{Neurological deficit score}

Functional deficits were assessed using an itemized neurologic deficit score prior to and 1, 7, 14, and 42 days after ICH surgery. The score is sensitive to deficits after striatal ICH and consists of five different sub-tests (9): spontaneous circling, bilateral forepaw grasp, contralateral hind limb retraction after lateral movement, contralateral forelimb flexion, and beam-walking ability. Scores on each sub-test were added to a total score ranging from 0 to 14 with higher scores indicating severer deficits. Animals were assessed in a blinded fashion.

\section{Quantification of lesion size and brain atrophy}

Brains were frozen using dry ice and sectioned at $40 \mu \mathrm{m}$ thickness using a sliding microtom (Leica Microsystems, Wetzlar, Germany). In order to assess the volume of the lesion, every sixth section was picked and stained with cresyl violet. The sections were examined using a microscope (Zeiss Axio Imager M2, Gottingen, Germany) equipped with a semiautomatic stereology system (Stereoinvestigator; MicroBright-Field, Colchester, VT, USA). Volumes of the affected hemispheres were quantified as: (average area of complete coronal section of the hemisphere - area of damage - ventricle $) \times$ interval between sections $\times$ number of sections (10).

\section{Immunostaining}

Free-floating sections were treated with $0.6 \% \mathrm{H}_{2} \mathrm{O}_{2}$ and $\mathrm{HCl}$ for 30 mins, blocked in $3 \%$ donkey serum with $0 \cdot 1 \%$ Triton X-100 and incubated with a primary antibody: (anti-BRDU, rat
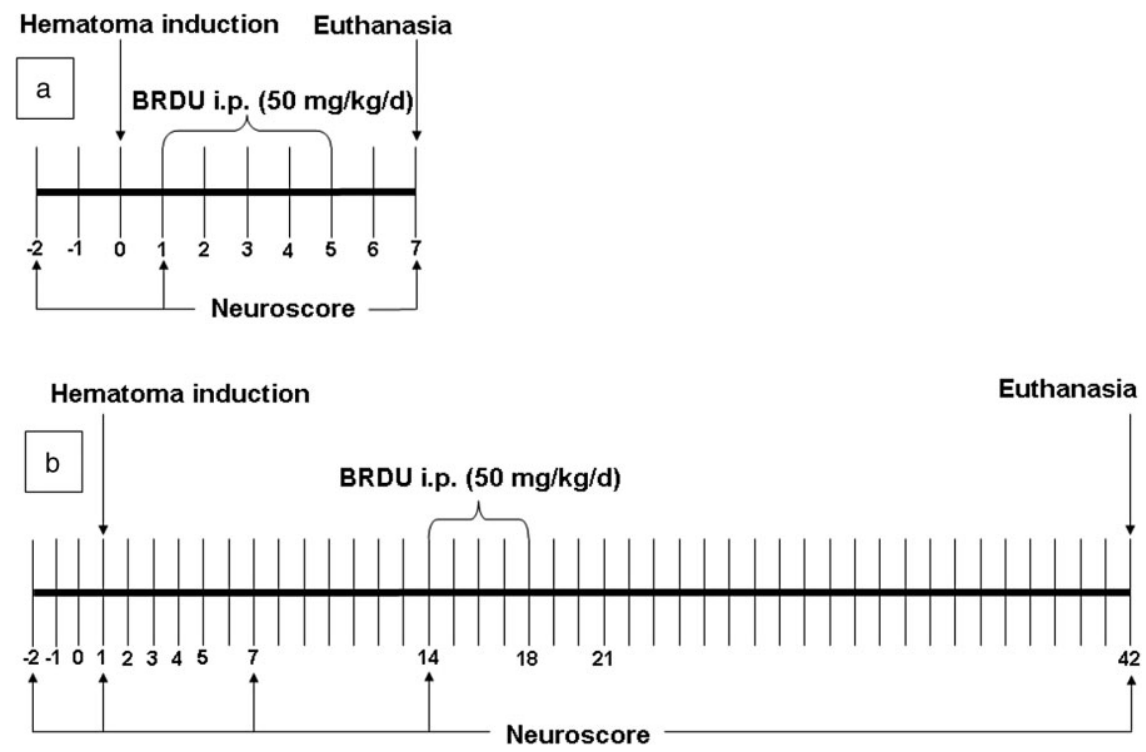

Fig. 1 Experimental design. To determine proliferation in both neurogenic niches, animals (a; $n=54)$ received BRDU (50 mg/kg) between day 1 to day 5 and were perfused on day 7 after ICH induction. To study survival, animals received BRDU for five consecutive days between day 14 and 18 (b; $n=46$ ) and were perfused on day 42 . 
monoclonal, 1:2000; AbD Serotec, Düsseldorf, Germany). Following washes with Tris-buffered saline (TBS), the antibody binding sites were visualized using a secondary antibody (donkey anti-rat, 1:1000, Dianova, Hamburg, Germany), avidin-biotinperoxidase complex (1:100), and 3,3 diaminobenzidine substrate kit (both Vector Laboratories, Burlingame, CA, USA). The specificity of the immunohistochemical reaction was assessed by replacing the primary antibody by TBS.

\section{Characterization and quantification of newborn cells}

Every sixth section $(240 \mu \mathrm{m}$ intervals) was selected from every brain, and the number of BRDU-positive cells in the hippocampal dentate gyrus (DG) and the sub-ventricular zone (SVZ) of both hemispheres was quantified following seven-days and 42 days after ICH. Labelled cells that intersected the uppermost focal plane or the lateral exclusion boundaries of the region of interest were not counted.

\section{Statistical analysis and power estimation}

Data were processed using the PASW STATISTICs 18 (SPSS Inc., Chicago, IL, USA) and the Office 2007 (Microsoft Corp., Redmond, WA, USA) software packages. After normality of distribution was confirmed using the Shapiro-Wilk and Kolmogorov-Smirnov tests, data were summarized as means and standard deviations and compared using the one-way analysis of variance with post hoc analysis. Neurologic deficit scores were reported by median and interquartile ranges (IQRs), and the Mann-Whitney $U$-test was used for comparison. The level of significance was set a priori at $P<0 \cdot 05$. The sample size was calculated to provide $80 \%$ power for the detection of $10 \%$ difference in lesion volume, provided a mortality of $<5 \%$. Endpoint variation was expected highest for systemic GCSF treatment, and sample size was adjusted to $n=30$.

\section{Results}

\section{GCSF treatment does not affect lesion size and brain atrophy}

Figure 2 shows representative coronary sections taken at day 7 and day 42 after ICH induction. At day 42, the ICH lesion in the right striatum was narrow and partially organized; the injured hemisphere was markedly shrunken with enlargement of the ipsilateral ventricle, volume loss of the striatum, and the cortical band. Volume of the lesioned hemisphere was significantly lower than the one of the contralateral side $(P<0.05)$. After sham procedure without blood injection, the volume of the lesioned hemisphere was significantly higher than in animals with blood injection but volumetry did not reveal any differences in lesion size or brain atrophy between animals in the active GCSF or saline groups (Fig. 2c,d).

\section{GCSF treatment does not affect proliferation in regions of adult neurogenesis}

In order to quantify cell proliferation in the SVZ and DG of the hippocampus, newborn cells were labelled with BRDU between day 1 and day 5 after ICH and quantified in the DG and the SVZ on day 7 (Fig. 3). Neither in the ipsilateal nor in the contraleteral hemisphere did systemic or local GCSF treatment significantly affect the number of BRDU-positive cells.
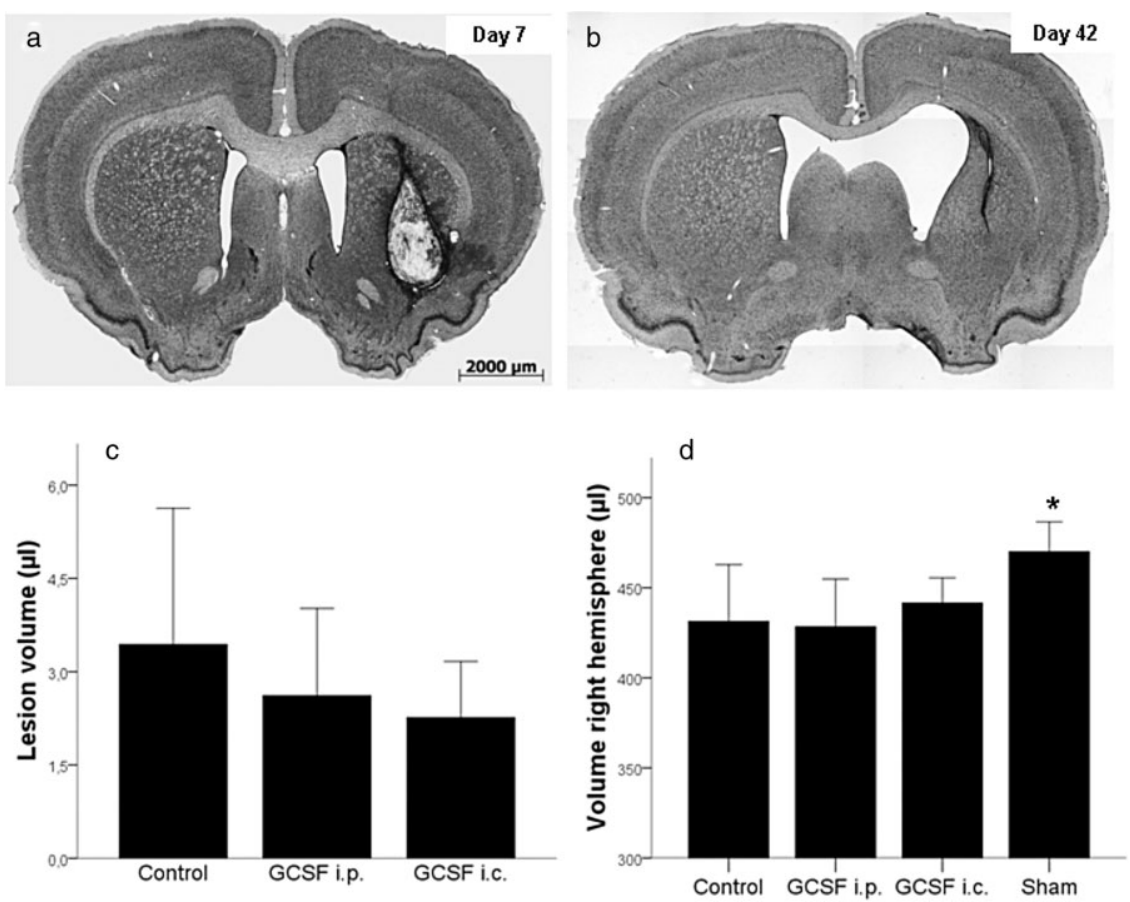

Fig. 2 Representative coronal staining of the anterior striatum for cresyl violet seven-days (a) and 42 days (b) after ICH. Note the organization of the hematoma, the hemispheric atrophy, and the enlargement of the side ventricle after six-weeks postlesioning. GCSF treatment did not affect lesion size (a) or the volume of the affected hemispheres (b) six-weeks after ICH induction $(* P<0.05)$. 

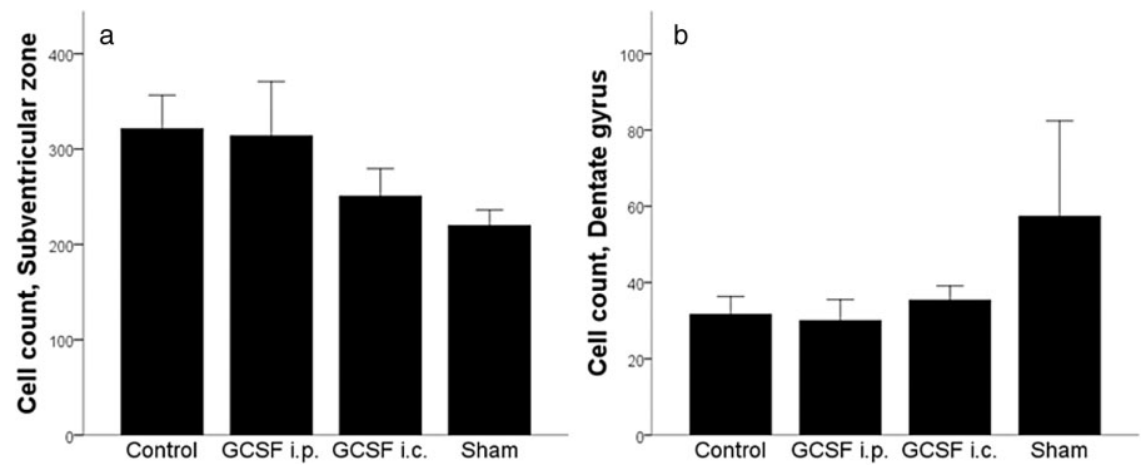

Fig. 3 BRDU enzyme-histochemistry seven-days after ICH lesioning. GCSF treatment did not alter the number of BRDU-positive cells in the subventricular zone (a) or the dentate gyrus (b).
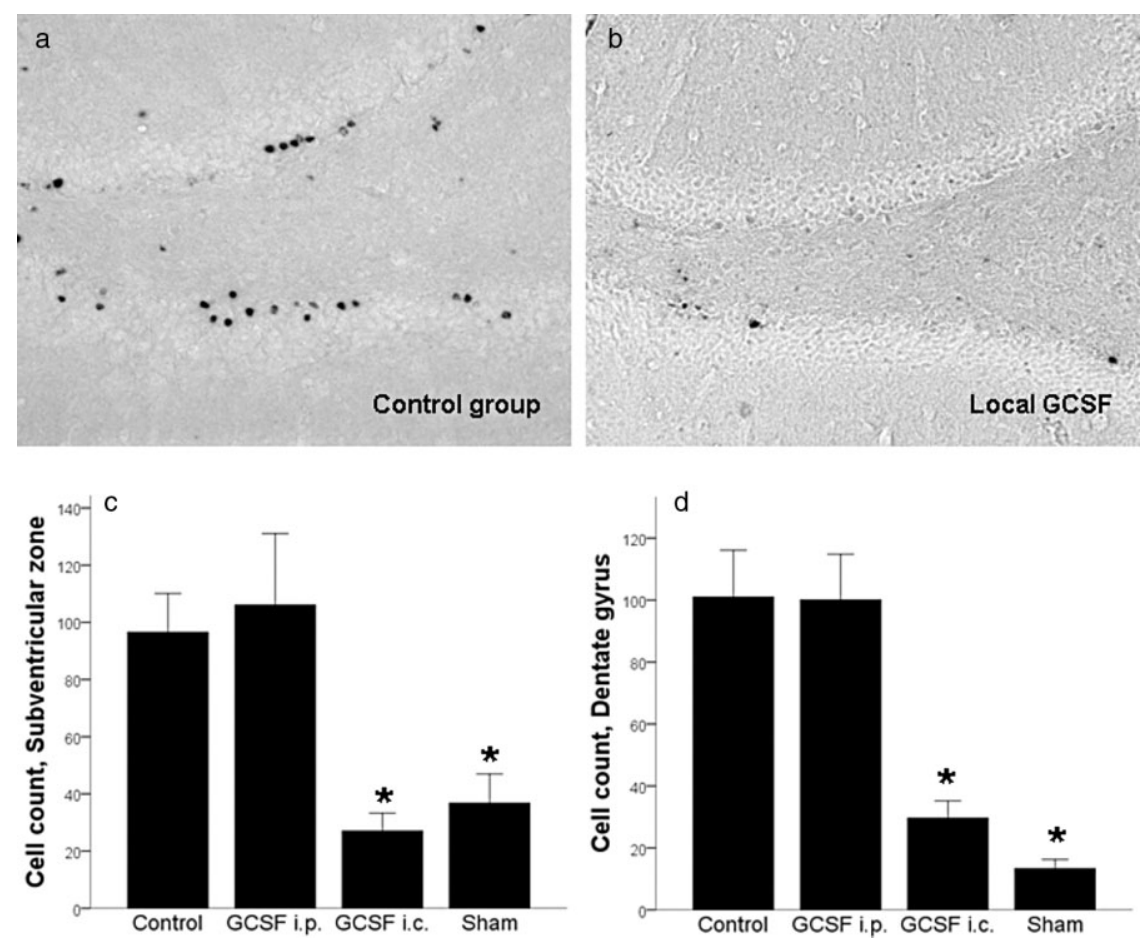

Fig. 4 Representative microphotographs of the dentate gyrus, taken 42 days after ICH induction (a: animal in the control group, b: animal after local GCSF application). Local GCSF treatment significantly reduced the number of BRDU-positive cells in the sub-ventricular zone (c) and the dentate gyrus (d) $(* P<0.05$, error bars indicate standard error of the mean).

\section{Local GCSF application decreases survival of newborn} cells in the SVZ and the DG

BRDU labelling was performed between day 14 and 18 , and cells were counted on day 42 to quantify cell survival after ICH lesioning. Sham procedure was associated with a significantly lower number of surviving cells within both neurogenic regions (compared with animals with ICH lesions. Systemic GCSF treatment had no significant effect on cell counts. Intracerebral application of GCSF resulted in a depletion of BRDU-positive cells to a level that was observed in animals after sham procedure (Fig. 4).

\section{Functional deficits}

Before ICH induction, all animals scored 0 on the NDS (Fig. 5). Four animals (4\%) died during or in the first hours after ICH surgery. On the first day following $\mathrm{ICH}$, all surviving animals showed mild to moderate functional deficits, reflected by a median NDS score of 4.0 (IQR 2.0-5.0). Differences between the three groups were statistically not significant. Animals in all groups underwent almost complete functional recovery until day 42. On day 14, animals with GCSF treatment showed a marginal advance in functional improvement, which was more distinct after local treatment.

\section{Discussion}

In this experimental study neither local nor systemic treatment with GCSF reduced lesion size or altered brain atrophy six-weeks post-ICH. Furthermore, there was no evidence for a proliferative 


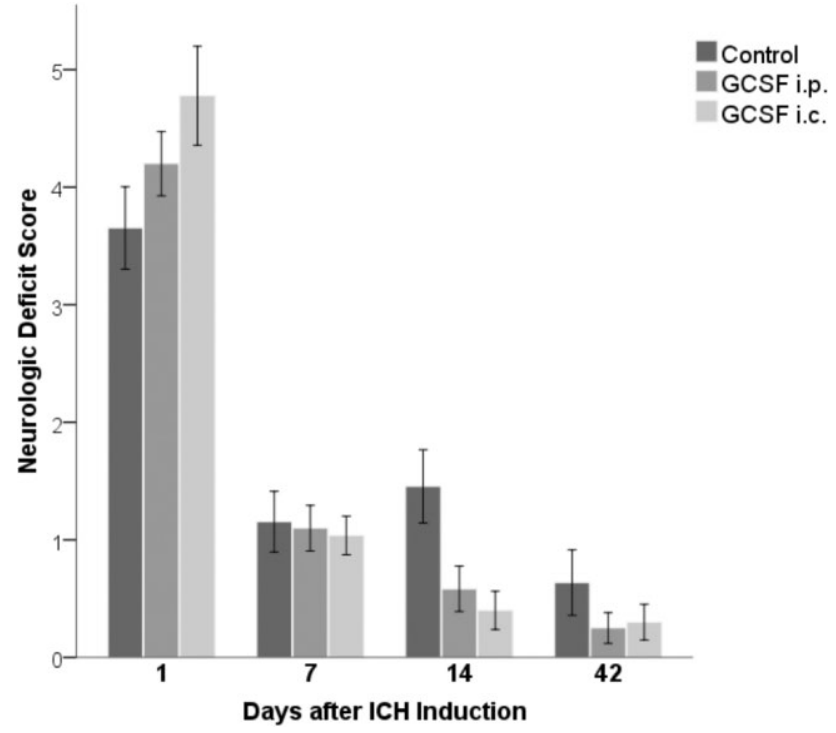

Fig. 5 Functional recovery from experimental $\mathrm{ICH}$. On the first day after ICH surgery, animals exhibited mild to moderate neurologic deficits. A subtle benefit from GCSF treatment was found in functional improvement at days 14 and 42 .

effect of GCSF on cerebral neurogenesis. The survival of cells in the DG and the SVZ was even reduced after local GCSF application.

Brain damage after ICH is attributed to primary and secondary mechanisms (11). Primary damage occurs early and is related to the mechanical force of hematoma expansion. Secondary damage is caused by products of hematoma degradation, which trigger perihemorrhagic inflammation, blood-brain-barrier disruption, and formation of cerebral edema during the following days and weeks after bleeding onset. Currently, the two best established and widely used animal models of ICH are the collagenase injection and the whole blood injection model, both of which cause different bleeding profiles, brain lesions, and levels of functional deficits. Both models are very limited in order to mimic the clinical condition (12), and it is general consensus that novel interventions should be tested in both experimental settings before being considered for clinical investigation. We therefore aimed to complement previous findings of GCSF treatment in collagenaseinduced ICH $(4,5)$ by applying GCSF to a second animal model in rodents. In our study, ICH surgery caused substantial brain damage and atrophy, reflected by a significant reduction in the volume of the injured hemisphere compared with the one after sham procedure. However, the lesion size and the brain volume were not affected by GCSF treatment. If any, differences in the degree of these parameters between active and control groups are supposed to occur to a marginal extent or beyond the first sixweeks after bleeding onset. However, in a recent study, functional outcome was improved by rehabilitation without evident changes in lesion volume (13), indicating that the volumetric brain damage does not necessarily predict functional impairment.

The SVZ and the hippocampus were repeatedly demonstrated to harbor regions of adult neurogenesis in rodents $(14,15)$. After cerebral ischemia, GCSF increased the number of newly generated neurons in the DG of rodents, and it was speculated whether this mechanism refers to enhanced structural and functional repair after stroke (3). However, GCSF did not promote cell proliferation at either site in our model of experimental ICH. In contrast, cell survival after six-weeks was even worsened after local growth factor application. Therefore, these results do not give evidence for a relevant impact of adult neurogenesis on functional recovery after $\mathrm{ICH}$.

Park et al. (4) reported that GCSF treatment attenuated sensomotor deficits with superior performance on the rotarod test and the modified limb-placing test, starting at day 14 after collagenase-induced $\mathrm{ICH}$ and persisting with increasing efficacy up to week 5. A similar result was reported by Zhang et al. (5), who observed significantly decreased impairment in the limb placement test at week 1 to week 4 following GCSF treatment. Compared with these reports, the results from neurologic outcome assessment were less clear in our study. Using an itemized neurological deficit score at repetitive time points, we observed spontaneous functional recovery in the majority of animals until day 42, irrespective of the study group. A small benefit from GCSF treatment was suggested at day 14 only. However, apart from haemorrhagic stroke, GCSF was reported to improve functional outcome in different animal models of neuronal damage, including cerebral ischemia (3), Parkinson's disease (16), Alzheimer's disease (17), amyotrophic lateral sclerosis (18), spinal cord injury (19), and retinal ganglion cell axotomy (20). Up to date, neither of these promising results could be transferred to clinical medicine, whereas GCSF recently failed to improve outcome in a large clinical trial on patients with ischemic stroke (21). Further experiments with complementary methods of outcome assessment are warranted to investigate, whether a constant benefit from GCSF treatment on functional recovery after ICH can be reproduced (9).

\section{Conclusion}

Granulocyte colony-stimulating factor treatment did not measurably reduce lesion size and brain atrophy at six-weeks following experimental ICH. There was no evidence for the promotion of cerebral neurogenesis by GCSF treatment, and a clear neuroprotective or neuroregenerative effect of GCSF could not be displayed. Subtle benefits in functional recovery were observed at 14 days after ICH and require confirmation by future investigations.

\section{References}

1 Morgenstern LB, Hemphill JC, 3rd, Anderson C et al. Guidelines for the management of spontaneous intracerebral hemorrhage: a guideline for healthcare professionals from the American Heart Association/American Stroke Association. Stroke 2010; 41:2108-29.

2 Hwang BY, Appelboom G, Ayer A et al. Advances in neuroprotective strategies: potential therapies for intracerebral hemorrhage. Cerebrovasc Dis 2011; 31:211-22.

3 Schneider A, Kruger C, Steigleder T et al. The hematopoietic factor G-CSF is a neuronal ligand that counteracts programmed cell death and drives neurogenesis. J Clin Invest 2005; 115:2083-98.

4 Park HK, Chu K, Lee ST et al. Granulocyte colony-stimulating factor induces sensorimotor recovery in intracerebral hemorrhage. Brain Res 2005; 1041:125-31. 
5 Zhang L, Shu XJ, Zhou HY et al. Protective effect of granulocyte colony-stimulating factor on intracerebral hemorrhage in rat. Neurochem Res 2009; 34:1317-23.

6 Shyu WC, Lin SZ, Yang HI et al. Functional recovery of stroke rats induced by granulocyte colony-stimulating factor-stimulated stem cells. Circulation 2004; 110:1847-54.

7 Kawada H, Takizawa S, Takanashi T et al. Administration of hematopoietic cytokines in the subacute phase after cerebral infarction is effective for functional recovery facilitating proliferation of intrinsic neural stem/progenitor cells and transition of bone marrow-derived neuronal cells. Circulation 2006; 113:701-10.

8 Bullock R, Mendelow AD, Teasdale GM, Graham DI. Intracranial haemorrhage induced at arterial pressure in the rat. Part 1: description of technique, ICP changes and neuropathological findings. Neurol Res 1984; 6:184-8.

9 MacLellan CL, Langdon KD, Churchill KP, Granter-Button S, Corbett D. Assessing cognitive function after intracerebral hemorrhage in rats. Behav Brain Res 2009; 198:321-8.

10 MacLellan CL, Davies LM, Fingas MS, Colbourne F. The influence of hypothermia on outcome after intracerebral hemorrhage in rats. Stroke 2006; 37:1266-70.

11 Xi G, Keep RF, Hoff JT. Mechanisms of brain injury after intracerebral haemorrhage. Lancet Neurol 2006; 5:53-63.

12 Kirkman MA, Allan SM, Parry-Jones AR. Experimental intracerebral hemorrhage: avoiding pitfalls in translational research. J Cereb Blood Flow Metab 2011; 31:2135-51.

13 MacLellan CL, Plummer N, Silasi G, Auriat AM, Colbourne F. Rehabilitation promotes recovery after whole blood-induced intracerebral hemorrhage in rats. Neurorehabil Neural Repair 2011; 25:477-83.

14 Kirschenbaum B, Doetsch F, Lois C, Alvarez-Buylla A. Adult subventricular zone neuronal precursors continue to proliferate and migrate in the absence of the olfactory bulb. J Neurosci 1999; 19:2171-80.

15 Arvidsson A, Collin T, Kirik D, Kokaia Z, Lindvall O. Neuronal replacement from endogenous precursors in the adult brain after stroke. Nat Med 2002; 8:963-70.

16 Frank T, Klinker F, Falkenburger BH et al. Pegylated granulocyte colony-stimulating factor conveys long-term neuroprotection and improves functional outcome in a model of Parkinson's disease. Brain 2012; 135(Pt 6):1914-25.

17 Tsai KJ, Tsai YC, Shen CK. G-CSF rescues the memory impairment of animal models of Alzheimer's disease. J Exp Med 2007; 204:127380.

18 Pollari E, Savchenko E, Jaronen M et al. Granulocyte colony stimulating factor attenuates inflammation in a mouse model of amyotrophic lateral sclerosis. J Neuroinflammation 2011; 8:74.

19 Dittgen T, Pitzer C, Plaas C et al. Granulocyte-colony stimulating factor (G-CSF) improves motor recovery in the rat impactor model for spinal cord injury. PLOS ONE 2012; 7:e29880.

20 Frank T, Schlachetzki JC, Goricke B et al. Both systemic and local application of granulocyte-colony stimulating factor (G-CSF) is neuroprotective after retinal ganglion cell axotomy. BMC Neurosci 2009; 10:49.

21 Ringelstein EB, Thijs V, Norrving B et al. Granulocyte colonystimulating factor in patients with acute ischemic stroke: results of the AX200 for ischemic stroke trial. Stroke 2013; 44:2681-7. 